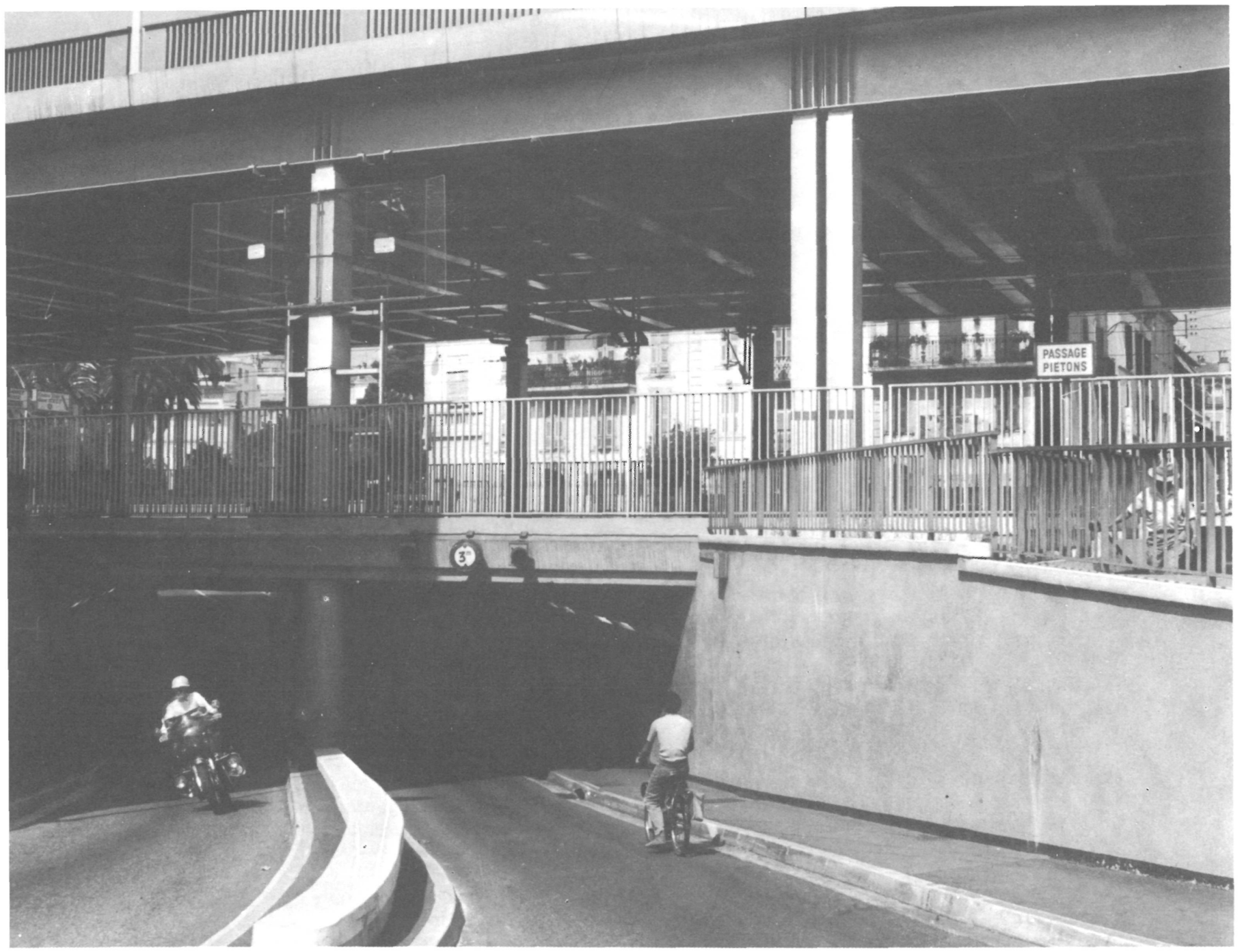

sinopsis

Se describe en este artículo la construcción, en Cannes, de la tercera trucción, en Cannes, de la tercera fase de un paso elevado realizado sobre andenes $y$ vias de ferrocarril, con el que se consigue eliminar una serie de curvas de la carretera cosfico en la ciudad.

Para este tercer tramo se eligió la construcción a base de estructura metálica, a pesar de que los dos tramos anteriores eran de hormigón. Se construyeron 15 vanos, de los cuales 14 de ellos llevan un emparrillado de vigas soldadas con losas de hormigón armado y el otro es un emparrillado de vigas laminadas sobre hormigón.

El total de acero utilizado fue de $3.360 \mathrm{t}$ sin contar las armaduras de los cimientos y de los tableros.

\section{Paso elevado sobre el ferrocarril, en Cannes \\ Francia}

Klaus Idelberger, Dipl. ingeniero

562-151

Nombres de ciudades como Toulon, St. Tropez, St. Ráphael, Cannes, Montecarlo, San Remo, Ventimiglia nos recuerdan el tiempo alegre de las vacaciones a las orillas del mar Mediterráneo, pero asustan por la gran cantidad de tráfico que existe en sus carreteras, especialmente en la carretera costera RN 7, a su paso por estas ciudades.

Sin embargo, la ciudad de Cannes:

- se libró en gran medida del tráfico internacional por la carretera de la costa;

- se realizaó, en la RN 7, un paso elevado de 4 a 6 carriles y $2 \mathrm{~km}$ de longitud, por encima de la estación de ferrocarril y a través de la ciudad moderna. Se salvaron de este 


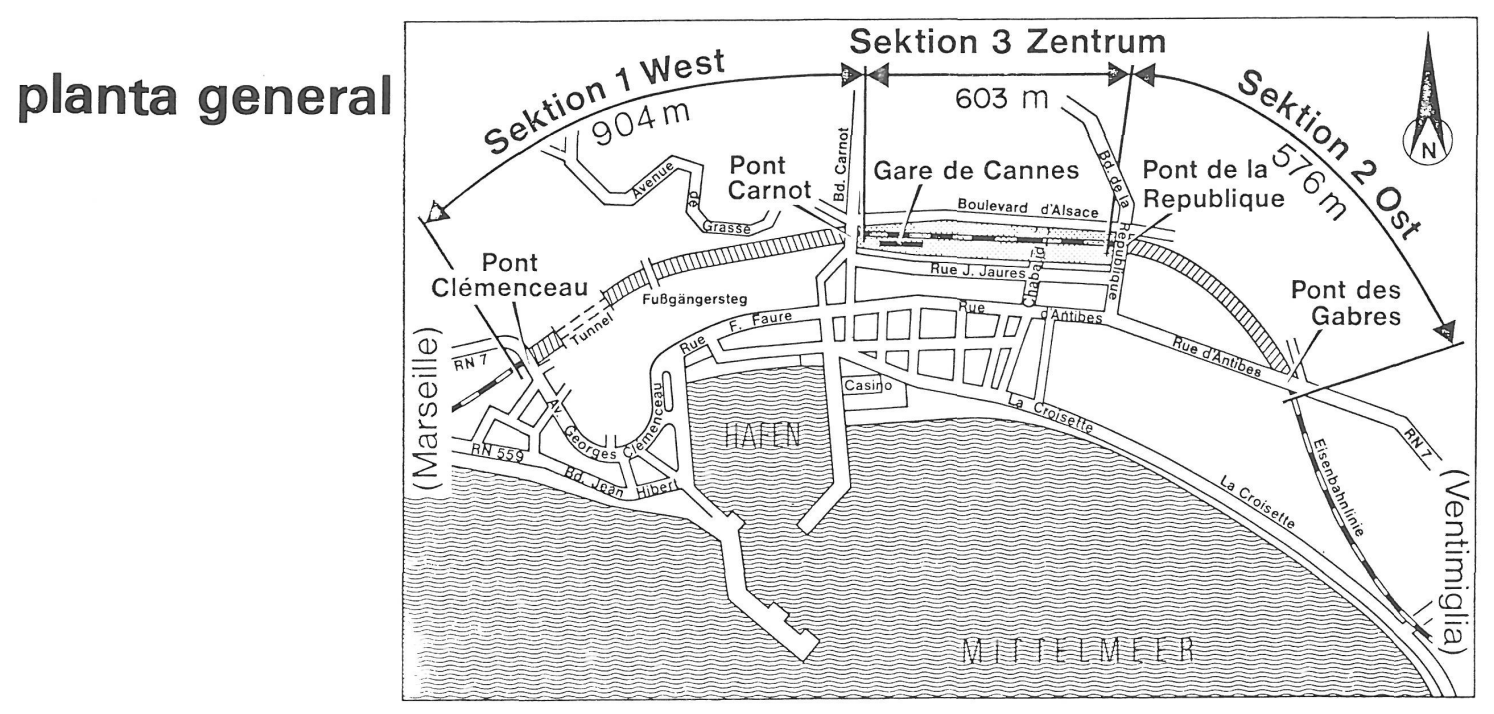

modo $3,5 \mathrm{~km}$ de curvas en la maravillosa carretera costera, entre la ciudad antigua y el puerto, así como el fotogénico paseo de palmeras "Croisette" entre los hoteles y la playa, zona en la cual los turistas dejan muchas divisas.

El paso elevado está formado por tres tramos en dirección oeste-este, de distintas longitudes, realizado cada uno de ellos en diferentes fases constructivas y en distintos materiales de construcción.

Los trazados de la carretera y el ferrocarril, que siguen el recorrido costero de la Cote d'Azur, sirvieron para la construcción de este paso elevado, ya que la RN 7 en su trazado anterior cruzaba la línea de ferrocarril dos veces en un recorrido de $3,5 \mathrm{~km}$ : a la entrada, por el puente de Clemenceau, en el lado oeste de la ciudad y a la salida, por el puente de Cabres, en el lado este.

Entre estos dos puntos se extiende el nuevo paso elevado de la RN 7 con el que se consigue acortar el recorrido en $2 \mathrm{~km}, \mathrm{y}$, a la vez, no afectar ni atravesar el casco antiguo de la ciudad. En primer lugar se realizó el tramó 1 , que va sobre la trinchera de la línea de ferrocarril, desde el puente Clemenceau, hasta el puente de Carnot situado al oeste de la estación. Con este tramo se saneó el tráfico del barrio "Le Suquet». Después se construyó el tramo 2 desde el puente de la República, al este de la estación, hasta el puente de Cabres. Para terminar la regulación del tráfico y entorpecer al mínimo su paso por la ciudad se realizó finalmente el tramo 3, que es el que describimos en este artículo.

\section{Paso elevado de acero que sirve a la vez como cubierta de andén}

Lo primero que se hizo, con el fin de sanear el tráfico, fue trasladar la estación de mercancías desde el centro de la ciudad hasta las afueras. Los dos primeros tramos del paso elevado comenzaron a realizarse antes de la electrificación del ferrocarril hacia 1967, mientras que la tercera y última fase constructiva se realizó desde febrero de 1972 hasta junio de 1974. Simultánea y paralelamente al paso elevado se construyeron un aparcamiento y un nuevo edificio de recepción, con lo que se consiguió que una estación antigua se convierta en una estación completa conteniendo todo el movimiento que genera el ferrocarril.

\section{CARACTERISTICAS DEL PASO ELEVADO}

\begin{tabular}{|c|c|c|c|c|c|}
\hline Tramo & Sobre & Hasta & Longitud & Terminación & Material \\
\hline 1 Oeste & Pont Clemenceau & Pont Carnot & 904 m & 1965 & Hormigón \\
\hline 2 Centro & Gare des Cannes & & $603 \mathrm{~m}$ & 1974 & Acero \\
\hline \multirow[t]{2}{*}{3 Este } & \multirow{2}{*}{$\begin{array}{l}\text { Pont de la } \\
\text { Republique }\end{array}$} & \multirow{2}{*}{$\begin{array}{l}\text { Pont des } \\
\text { Cabres }\end{array}$} & $576 \mathrm{~m}$ & 1967 & Hormigón \\
\hline & & & $2.083 \mathrm{~m}$ & & \\
\hline
\end{tabular}


Aunque entre las soluciones propuestas estaba la de la construcción con hormigón pretensado, las autoridades municipales y las del farrocarril del Estado eligieron una estructura metálica para el nuevo paso elevado, que sirve de andén al mismo tiempo. Esta solución es la óptima desde el punto de vista del costo, del urbanismo, de la arquitectura y de la organización funcional de una gran obra.

Se levantaron 2 vanos de transición entre los antiguos tramos de hormigón y el tercer tramo más moderno de acero, y entre ellos se construyó el paso elevado, dividido en 14 vanos de vigas soldadas de acero con tablero de hormigón armado, todo ello apoyado sobre pilares de acero soldados. En total se utilizaron 3.360 t de acero, sin incluir las armaduras.

Las secciones transversales presentan, a fin de facilitar el desagüe, una inclinación del 2 al 3 por ciento.

La sección longitudinal muestra una pequeña curvatura. Respecto al nivel del mar, la calzada tiene en el extremo este una altura de $14,5 \mathrm{~m}$, a continuación sube casi imperceptiblemente en la zona central hasta los $15,3 \mathrm{~m}$ de altura $\mathrm{y}$, finalmente, baja de nuevo en el extremo oeste a $13,4 \mathrm{~m}$ ó $12 \mathrm{~m}$ de altura. Estas cotas vienen determinadas por la existencia de puntos obligados como, por ejemplo, la entrada y salida de carreteras contiguas, la situación de las vías y las distancias de seguridad.

En planta, el paso elevado presenta un perfil ligeramente alabeado. Desde el extremo este, durante unos $300 \mathrm{~m}$, el trazado va en línea recta, girando después $1,36^{\circ}$ hacia el sur y siguiendo nuevamente en línea recta unos $100 \mathrm{~m}$ hasta el extremo oeste.

Los apoyos en el lado sur discurren a lo largo del aparcamiento y del edificio de recepción y, en la parte norte, junto al Boulevard d'Alsace, en forma de línea recta poligonal. Debido tanto a un ensanchamiento del paso elevado, frente al edificio de recepción, como al giro ya mencionado de $1,36^{\circ}$, los 50 puntos de apoyo tienen en algunos puntos una distancia entre ejes algo mayor.

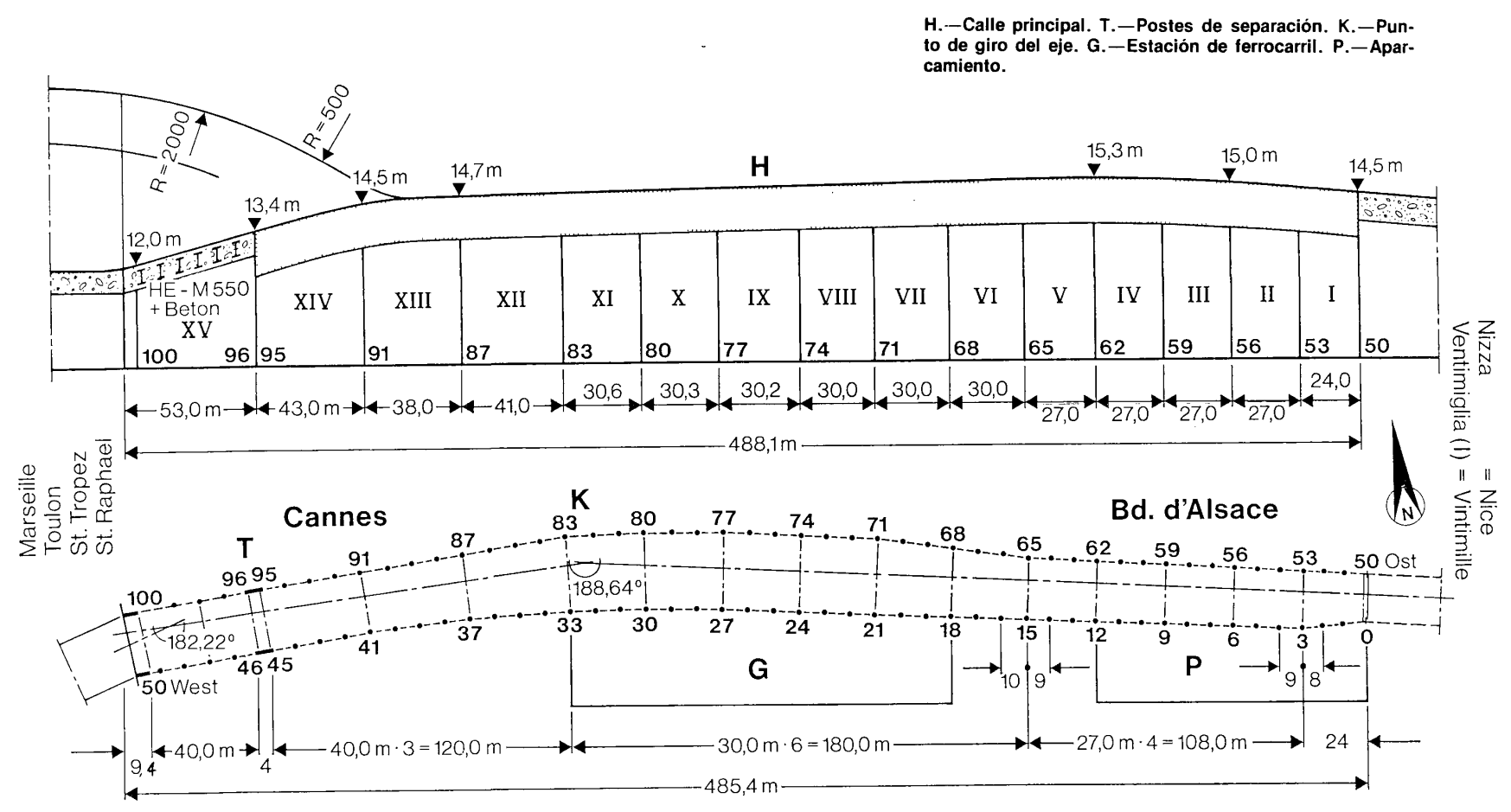

puentes zona 3 
secciones
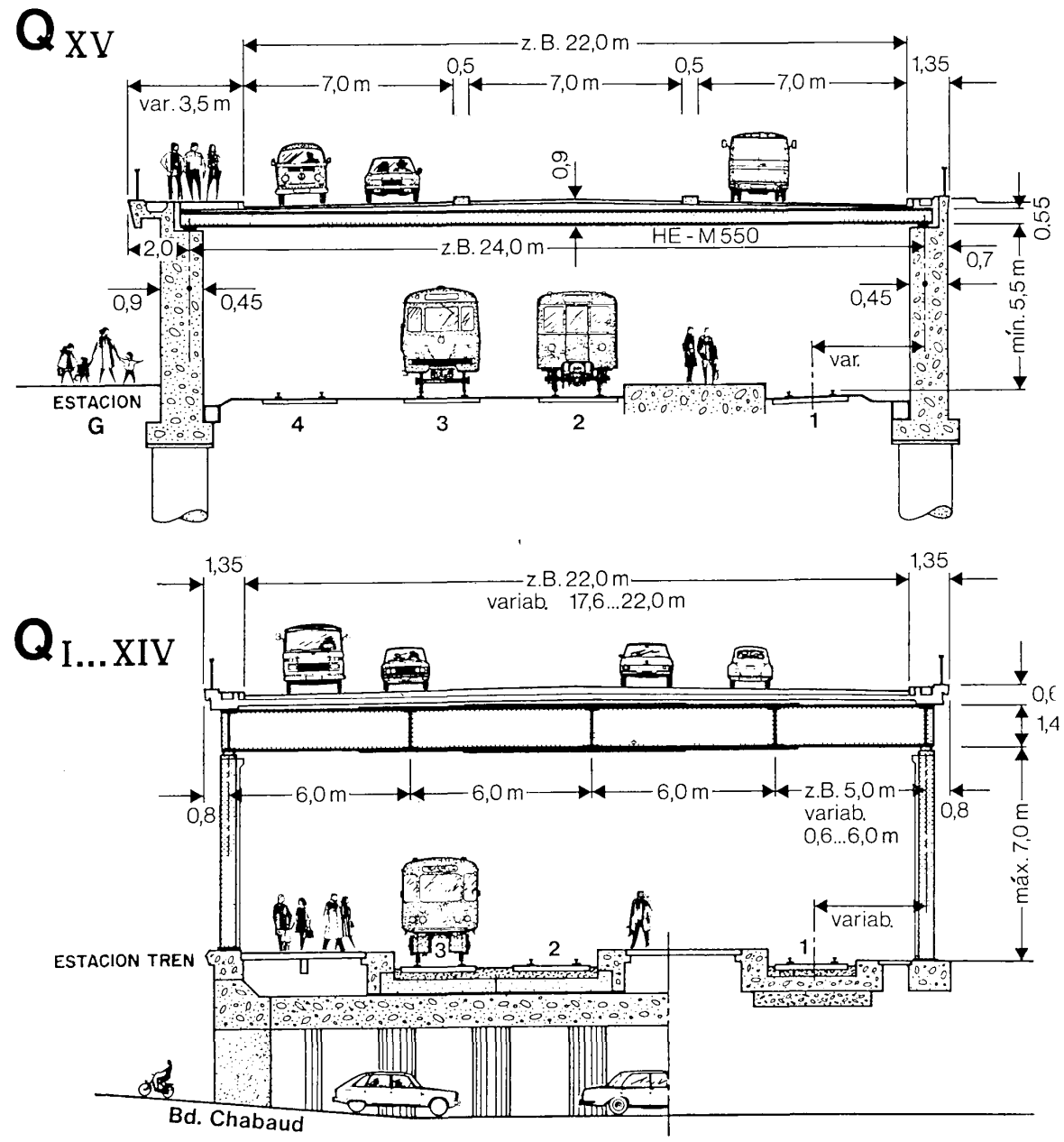

La solución adoptada es la de 15 vanos entre los dos estribos y 14 juntas de dilatación. Las juntas de dilatación están situadas sobre 13 soportes dobles y un pilar en doble $T$ (este último cerca del extremo oeste). Los 15 vanos tienen una longitud, entre ellos, de hasta $40 \mathrm{~m}$, y una sección transversal de hasta $30 \mathrm{~m}$; a ello se suman además algunos decímetros en voladizo a ambos lados de los tableros. El paso elevado se divide por tanto en la dirección del avance constructivo de la siguiente manera:

\begin{tabular}{|c|c|c|c|c|c|}
\hline Tramos & & gitud del tablero $(\mathrm{m})$ & Longitud total $(\mathrm{m})$ & Anchura del tablero $(\mathrm{m})$ & Tipo de estructura \\
\hline $\begin{array}{l}\text { I Este } \\
\text { II hasta V } \\
\text { VI hasta XI } \\
\text { XII hasta XIV }\end{array}$ & $\begin{array}{l}1 \\
4 \\
6 \\
3\end{array}$ & $\begin{array}{l}3 \times 8=24 \\
3 \times 9=27 \\
3 \times 10=30 \\
4 \times 10=40\end{array}$ & $\begin{array}{r}24,0 \\
108,0 \\
180,0 \\
120,0\end{array}$ & $\begin{array}{l}18,6 \ldots .22,6 \\
22,6 \ldots .24,0 \\
24,0 \ldots .30,0 \\
30,0 \ldots .24,0\end{array}$ & $\begin{array}{l}\text { Emparrillado de vigas } \\
\text { soldadas con pla- } \\
\text { ca de hormigón } \\
\text { armado. }\end{array}$ \\
\hline $\begin{array}{l}\text { Pilar de separación } \\
\text { XV Oeste } \\
\text { Estribos }\end{array}$ & $\begin{array}{l}0 \\
1 \\
0\end{array}$ & $\begin{array}{c}4 \times 10=40 \\
(5,4+4)\end{array}$ & $\begin{array}{r}4,0 \\
40,0 \\
9,4\end{array}$ & $\begin{array}{c}24,0 \\
24,0 \ldots 24,0 \\
24,0\end{array}$ & $\begin{array}{l}\text { Emparrillado de vi- } \\
\text { gas laminadas en } \\
\text { lecho de hormigón. }\end{array}$ \\
\hline
\end{tabular}


El tablero del vano XV, que es el vano de enlace con los tramos anteriormente construidos, es de emparrillado de vigas laminadas en lecho de hormigón. Los vanos I hasta XIV descansan, por el contrario, sobre un emparrillado de vigas soldadas.

\section{Tramo con emparrillado de vigas laminadas sobre lecho de hormigón}

Los tableros del vano XV constan de una capa de hormigón in situ, con un espesor máximo de 0,9 metros, vertido sobre perfiles de acero laminado. Estas vigas transversales están colocadas a una distancia de 0,55 $\mathrm{m}$ y tienen una luz de $24 \mathrm{~m}$, como máximo. Las vigas transversales son perfiles laminados, normalizados en I de la serie reforzada HE-M, con simetría central en la mayoría de los casos, reforzados incluso con platabandas adicionales y provistos siempre de una sobreelevación que alcanza algunos centímetros. Esta sobreelevación compensa la flexión bajo un cuarto de carga de circulación máxima admitida, así como bajo la carga propia total. (Peso del hormigón + el del acero + el de los conductos + el de las barandillas.)

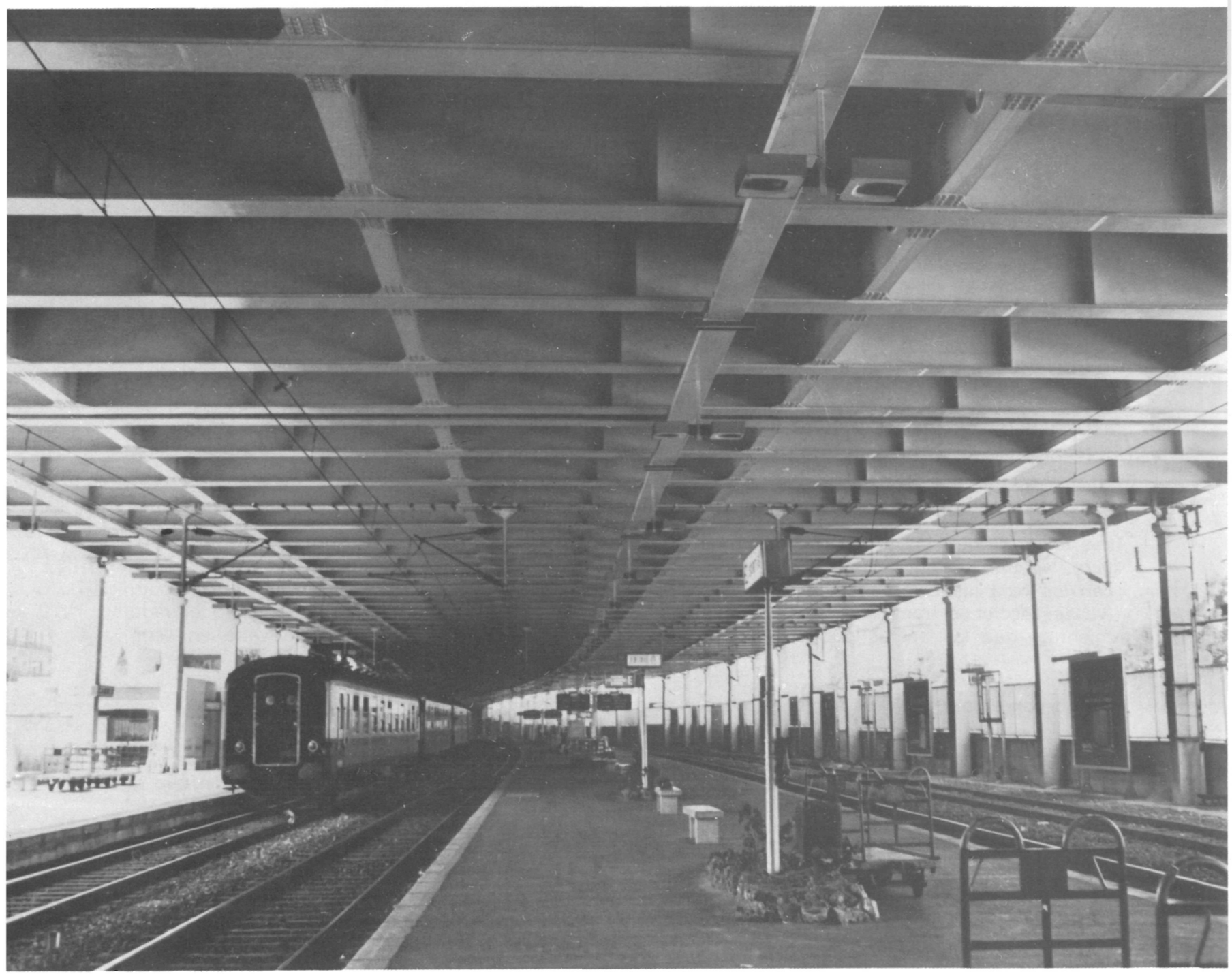




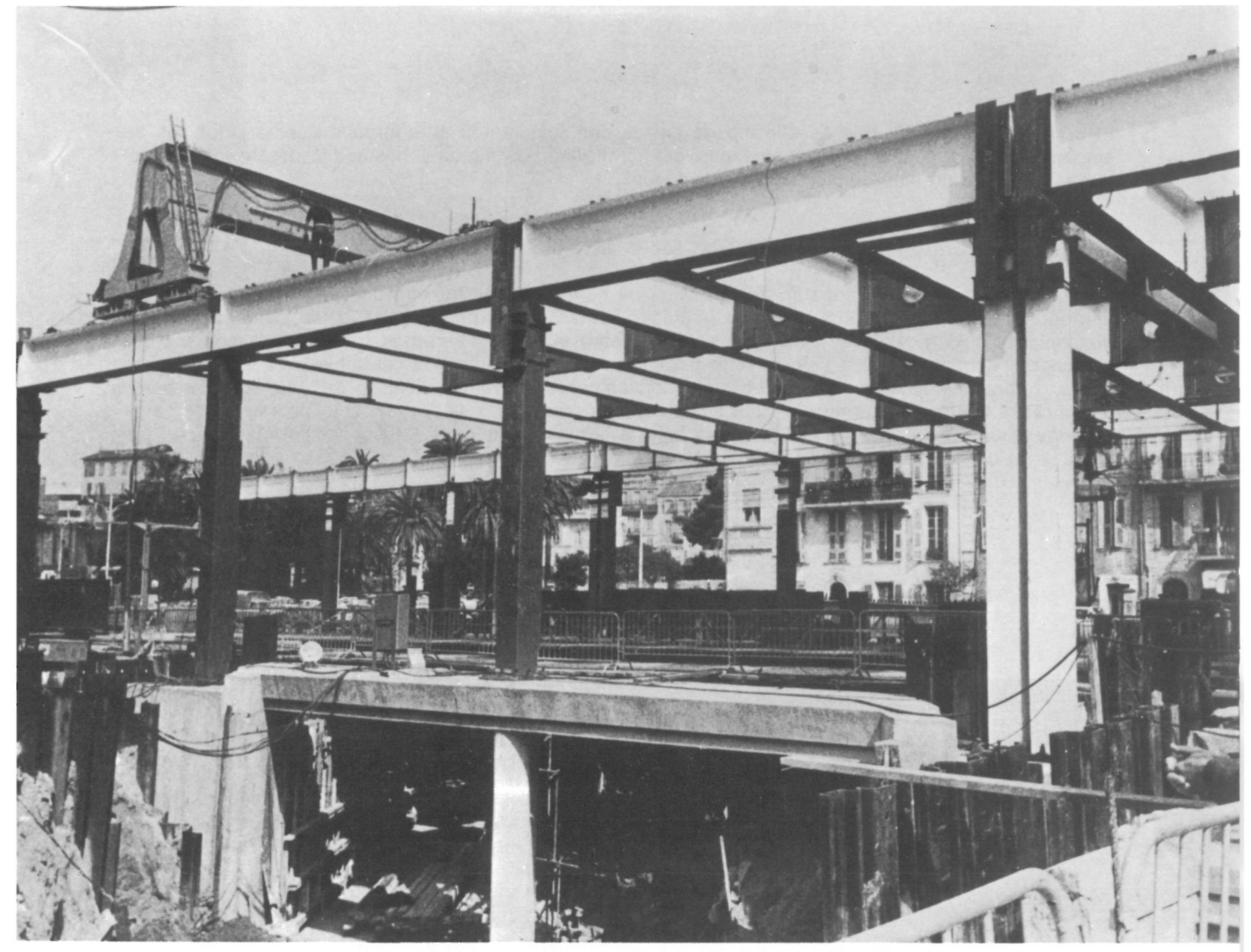

Chapas longitudinales acoplan entre sí las vigas transversales y las aseguran contra pandeo. Estas chapas poseen unos agujeros en el alma para los conductos de todo tipo. Para el sostenimiento de este emparrillado sirven, para los vanos, los soportes de acero que más adelante se describirán. Sin embargo, si el sostenimiento lo consideramos por tramos se utilizan discos de pared de hormigón armado de una anchura máxima de $1,35 \mathrm{~m}$. Cojinetes de neopreno transmiten las cargas y permiten dilataciones y acortamientos cuando se producen cambios de temperatura.

Las ranuras entre los apoyos y los vanos, asi como entre vanos van provistas de juntas dilatables pero, además, y por razones de seguridad, van dotados también de un canal para desagüe. Los canales para la lluvia han sido dispuestos a lo largo del paso elevado y de las vías del ferrocarril (véase secciones transversales). Existen arcenes de emergencia a todo lo largo del paso elevado, mientras que las aceras públicas y carreteras de enlace sólo van colocadas de vano en vano.

\section{Tramo con emparrillado de vigas soldadas y placa de hormigón armado}

Los tableros de hormigón armado de los vanos I hasta XIV están formados por hormigón in situ de 0,2 $\mathrm{m}$ de espesor, como mínimo, vertido con ayuda de elementos prefabricados de hormigón que se habían encajado antes como finos tableros encofrados en el retículo de los cordones superiores del emparrillado, habiéndose tendido también capas de armadura. Esta placa de hormigón armado se encuentra unida al emparrillado con angulares de acero.

Estas vigas transversales tienen una luz de $30 \mathrm{~m}$ como máximo, a una distancia de 3 a $3,33 \mathrm{~m}$ (en el vano I hay una distancia especial de $2,66 \mathrm{~m}$ ). Las vigas transversales son perfiles de alma llena de $1,40 \mathrm{~m}$ de altura constructiva nominal, provistos en su parte central de láminas de refuerzo y con sobreelevación. 
Chapas longitudinales acoplan entre sí las vigas transversales - generalmente tres filas a una distancia de $6 \mathrm{~m}$-, aunque en el caso excepcional de la existencia de una cuarta via frente al edificio de recepción (vía de depósito) se colocan cuatro filas. Son perfiles soldados en I de alma llena, con una altura nominal de $1,40 \mathrm{~m}$ y la mayor parte de las veces con un "agujero de hombre», de forma circular, para conductos. Estas chapas van atornilladas como mecanismo de seguridad contra pandeo y a efectos de una nivelación de carga entre las vigas transversales, cuyos extremos se encuentran sobre soportes longitudinales.

Las vigas longitudinales se extienden a ambos lados del paso elevado y del andén a una distancia de $18,6 \mathrm{~m}$ hasta $24,0 \mathrm{~m}$. Son perfiles soldados en I de alma llena, con una altura de 1,40 $\mathrm{m}$ y con rigidizadores sólo sobre los apoyos.

Los apoyos tienen, incluyendo sus bases que en algunas partes se encuentran hundidas, una altura de $5,50 \mathrm{~m}$ hasta $7 \mathrm{~m}$. Los apoyos son perfiles-cajón soldados de 0,50 m de canto. Sus bases están ancladas, y sus cabezas llevan dispositivos para la nivelación y para la dilatación: Cuatro cojinetes móviles con forma de rodillos de acero moldeados en las cuatro esquinas de los vanos del paso elevado y entre ellos dos o cuatro cojinetes fijos en lados opuestos.

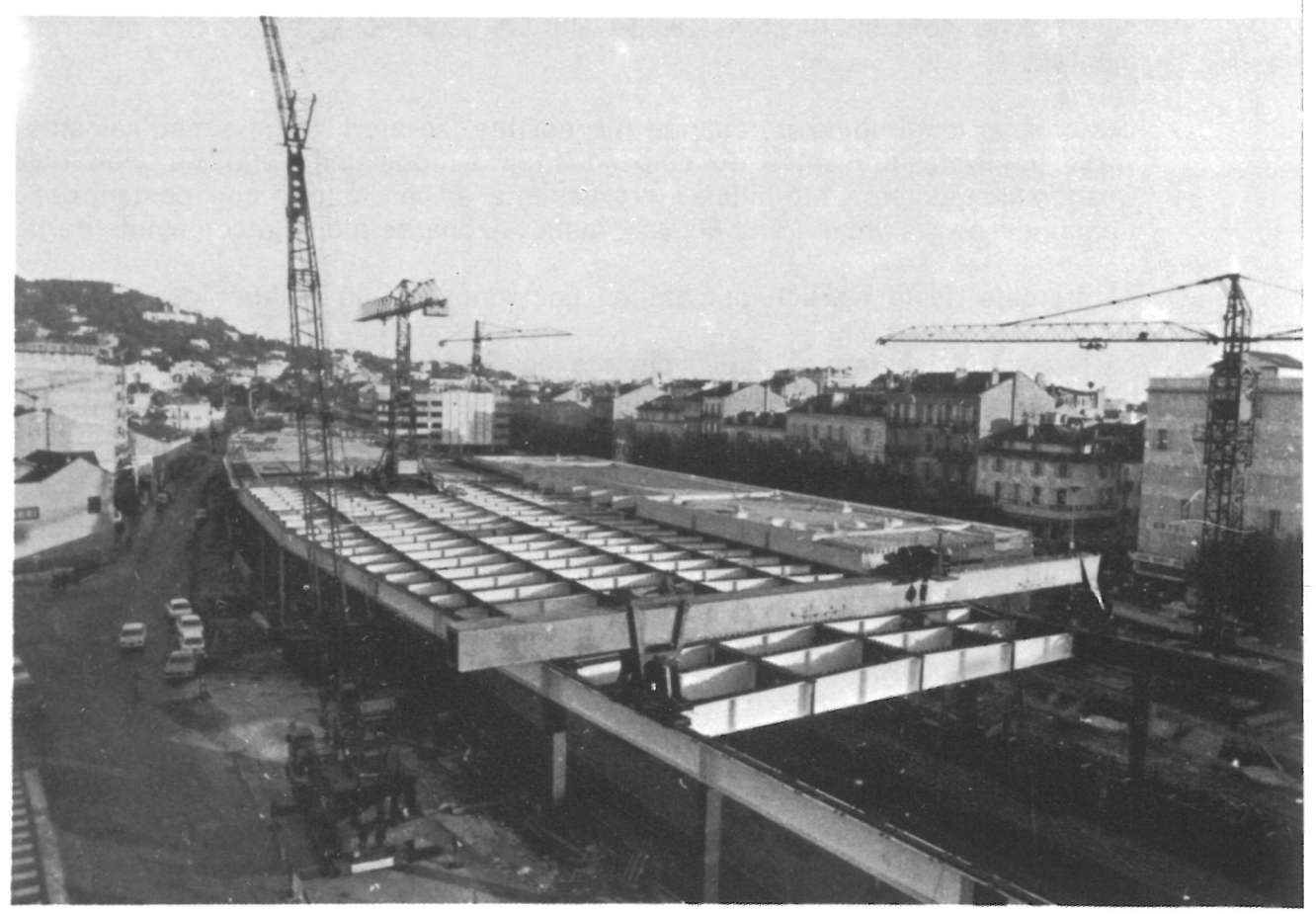

FOTOS: IDELBERGER, y J. RICHARD-DUCROS

\section{El avance en voladizo sobre las vías no constituye ningún problema}

Aun cuando se utilizó también la reconstrucción de la estación, para corregir las vías, debieron seguir funcionando en forma permanente al menos dos vias y dos andenes del tramo VentimigliaMarsella.

En primer lugar se colocaron los apoyos sobre zapatas de hormigón situadas aproximadamente al nivel de las vías, y allí se fijaron de forma provisional con anclajes de tensión previa. 
En segundo lugar se situaron las vigas longitudinales sobre la parte superior de los apoyos, y alli se fijaron de forma provisional. Estas vigas Iongitudinales se suministraron, cuando fue posible, con la longitud de un vano completo del paso elevado y se encajaron rápidamente. En caso de que el vano fuera demasiado grande se ensamblaban a nivel de tierra varios elementos hasta alcanzar la longitud del vano y después se encajaban. Sólo en casos excepcionales se unian las vigas arriba, sobre la parte superior de los apoyos. Para las juntas se utilizó la soldadura en aquellas zonas que iban a ser visibles posteriormente, y tornillos RV en aquellas otras zonas destinadas a ser cubiertas por el edificio de la estación o por los muros de los extremos.

Los soportes y vigas longitudinales de la parte sur, que da al nuevo edificio de recepción, se transportaron hacia alli con vagonetas de ferrocarril y se elevaron mediante una grúa móvil. Esta grúa circulaba sobre terrenos de los ferrocarriles del Estado SNCF, en concreto sobre andenes o bien sobre un emparrillado provisional situado sobre las vías. Los soportes y vigas longitudinales de la parte norte se transportaron con vehículos de carga y se trasladaron con una grúa sobre camión que circulaba sobre el Boulevard d'Alsace.

En tercer lugar, inmediatamente después de situadas las vigas longitudinales, se realizó el montaje de las vigas transversales con ayuda de una grúa especial que circulaba sobre las vigas longitudinales. Esta grúa se diseñó especialmente para esta fase de la construcción. Todas las vigas transversales llegaron al Boulevard d'Alsace sobre plataformas bajas $y$, en seguida, se colocaron mediante un camión-grúa sobre los tramos del paso elevado, ya existentes, en la parte este de la nueva zona de construcción. Desde alli las remolcó la susodicha grúa móvil hasta el lugar de montaje.

Las chapas longitudinales, cuando fue posible, se atornillaron simétricamente a ambos lados de las vigas transversales, antes de colocarlas en su posición definitiva, con objeto de evitar vuelcos. Cuando no pudieron atornillarse previamente se las colocó con posterioridad, o sea, después del transporte de las vigas transversales hasta la zona de montaje con ayuda de la misma grúa móvil.

Al montaje de la estructura metálica por vanos siguió el hormigonado del tablero de la calzada.

La grúa móvil se fabricó especialmente para que pudiese realizar montajes sin ningún riesgo fuera del gálibo ferroviario y de sus conductores superiores, que se encuentran bajo tensión y que no siempre pudieron desconectarse. Se dimensionó para que pudiera cargar con suficiente seguridad las vigas transversales más pesadas, incluyendo las chapas longitudinales incorporadas. La grúa móvil estaba formada por una poderosa viga-cajón cuadrangular, con mecanismo de rodadura, rígidamente montado en su base así como uno desplazable lateralmente. El mecanismo de rodadura desplazable lateralmente abarcaba con una corredera la viga-cajón de la grúa de tal forma que la grúa móvil, con ancho de vía variable, podia adaptarse a la distancia creciente y decreciente de la viga longitudinal. La viga-cajón llevaba un carrito con un mecanismo elevador de precisión para depositar todas las piezas constructivas del paso elevado precisamente en su lugar correcto.

Puentes de montaje cubrieron la zona de las vías de tal manera que los materiales de construcción que caian desde el lugar de las obras no podian llegar a esta zona.

\subsection{0 t de acero (A-52) galvanizado y con protección de pintura anticorrosiva}

Se utilizaron las siguientes calidades y cantidades de acero, sin contar las armaduras de los cimientos y de los tableros de la calzada:

$2.700 \mathrm{t} \begin{cases}\text { Acero } 52-3 & \begin{array}{l}\text { Nervios, vigas transversales, chapas longitudinales, vigas longitudinales, } \\ \text { soportes. }\end{array} \\ \text { Acero } 52-1 & \text { Zonas de transición de la calzada. }\end{cases}$

$510 \mathrm{t}$ Acero 42 Cordones superiores de algunas vigas (ya que en las zonas de presión no responde un acero de alta calidad). Piezas de separación.

55 t Fundición 65 Instalaciones de reglaje de la altura y de la posición.

$55 \mathrm{t} \quad$ Tornillos RV. Anclajes.

$40 \mathrm{t} \quad$ Material menudo. 
Un revestimiento protector contra incendios se consideró innecesario a la vista de la existencia de las suficientes vias de escape y debido a la falta de cargas con peligro de incendio. En cuanto al servicio eléctrico no hay que temer generación de chispas por parte de locomotoras y automotores. Los modernos PKw sólo pueden incendiarse a la fuerza o, como se ha puesto de manifiesto, en los numerosos ensayos contra incendios realizados en aparcamientos.

Se usó como protección superficial:

$\star 200 \mu \mathrm{m}$. Metalización de cinc (galvanizado o cincado por proyección);

$\star$ una capa de Wash-Primer;

$\star$ dos capas de pintura anticorrosiva.

El paso elevado está iluminado, a todo lo largo, por algunos postes colocados en las calles y plazas contiguas, aunque la mayoría se han situado sobre el propio paso elevado.

Este artículo ha sido reproducido del Strasse und Tiefbau con permiso del Editor.

\section{résumé}

PASSAGE SURELEVE SUR LE CHEMIN DE FER A CANNES - FRANCE

Klaus Idelberger, ingénieur diplômé

Dans cet article l'auteur décrit l'exécution, à Cannes, du troisième tronçon d'un passage surélevé sur des quais et des vois de chemin de fer. II permet d'éliminer une série de courbes de la route cotiere et de faciliter, en plus, le trafic en ville.

Pour ce troisième tronçon, il a été choisi une structure métallique, malgré que les deux tronçons précédents alent été exécutés en béton. Ce troisième tronçon a 15 travées, dont 14 sont constituées par des poutres en treillis soudées à des dalles en béton armé et l'autre travée constituée par des poutres en treillis laminées sur béton.

Le total d'acier utilisé a été de $3.360 \mathrm{t}$ sans compter les armatures des fondations et des tabliers.

\section{summary}

OVERPASS BRIDGING RAILWAY TRACKS - CANNES - FRANCE

Klaus Idelberger, Dipl. Engineer

This article describes the construction in Cannes of the third section of an overpass bridging number of railway tracks and platforms, achieving the supression of many curves in the coastal highway and this without interfering with city traffic.

For this third section the construction method chosen was a metal structure, despite the fact that the two previous sections were built in concrete. This overpass section has 15 spans, 14 of them are solved with a grid of welded beams supporting reinforced concrete slabs while the remaining span is a grid of laminated beams on concrete.

The total amount of steel used was $3,360 \mathrm{Tm}$, aside from the foundations and decking reinforcements.

\section{zusammenfassung \\ EISENBAHNUEBERGANG IN CANNES - FRANKREICH \\ Klaus Idelberger, Dipl.-Ing.}

In diesem Artikel wird der Bau einer dritten Etappe eines Bahnüberganges über Nahnstelge und Gieise in Cannes beschrieben. Auf diese Weise werden mehrere Kurven der Küstenstrasse und eine Umgehung des Stadtverkehrs erzielt.

Für diese dritte Strecke wurde für den Bau eine Metallstruktur gewählt, obwohl die vorigen Strecken aus Beton hergestelit wurden. Man errichtete 15 Hohlräume, 14 davon weisen geschweisste Balkengitter mit Betonplatten auf, der restliche enthält Walzgitterbalken auf Beton.

Insgesamt fanden 3.360 t Stahl Verwendung, darin sind die Bewehrungen der Gründung und Platten nicht enthalten. 